\title{
Effets du comportement différé sur le creusement des tunnels
}

A. KLEINE

A. GIRAUD

Laego

École nationale supérieure de géologie rue du Doyen

Marcel-Roubault

54501 Vandouvre-lès-Nancy alexandra.kleine@edf.fr

F. LAIGLE

Centre d'ingénierie hydraulique EDF

Savoie Technolac 73373 Le Bourget-du-Lad Cedex
A travers le retour d'expérience sur le creusement du tunnel de Tartaiguille, dans les marnes de l'Aptien, cet article montre que la prise en compte du comportement différé est un facteur susceptible de justifier la réduction de la résistance de la roche in situ.

Un modèle élasto-visco-plastique est proposé d'un point de vue conceptuel et analytique. Il a été mis en œuvre dans le cadre de simulations numériques de l'excavation d'une galerie circulaire. Ses applications ont permis de mettre en évidence les effets de la viscosité, de la vitesse de creusement, de la pose d'un revêtement, et d'étudier le comportement à très long terme du massif.

Mots-clés : comportement mécanique différé, roches argileuses, ouvrages souterrains, modèle de comportement, viscoplasticité, vitesse de creusement, soutènement.

\section{Effects of the time-dependent behavior on underground tunnels}

Through the back analysis from Tartaiguille railway tunnel, driven in Aptian marls, this paper shows that the timedependent behavior may justify the decreasing of the rock masses strength in field.

An elasto-visco-plastic constitutive model is described, from a conceptual and analytical point of view. It was used for numerical simulations of a circular gallery excavation. Its applications highlight the effects of the viscosity, of the digging process rate, of a support and allow to study very long term behavior of the rock mass.

Key words: time-dependent behavior, argillaceous rocks, underground openings, constitutive model, viscoplasticity, loading rate, support structure. 


\section{Introduction}

Dans le domaine des travaux souterrains, il a depuis longtemps été observé que les caractéristiques mécaniques des géomatériaux varient en fonction de l'échelle d'étude et de mesure (Bieniawski, 1974). Ainsi, toutes conditions étant identiques par ailleurs, il est généralement observé que la résistance en compression diminue avec l'accroissement de la dimension de l'échantillon testé. Par extrapolation, il en découle que les caractéristiques mécaniques du massif à l'échelle de l'ouvrage sont a priori plus faibles que celles mesurées et déterminées en laboratoire à partir d'un échantillon centimétrique, Cette notion, illustrée par Hoek et al. (1994), sur la figure 1, traduit ce qui est couramment appelé « l'effet d'échelle $»$, qui attribue la baisse des caractéristiques mécaniques de la masse rocheuse à l'accroissement du taux de discontinuités et de défauts au sein de ce volume.

Si cette notion est prépondérante dans un grand nombre de contextes géologiques et de configurations d'ouvrages, elle ne peut être suffisante pour justifier certaines observations et certains retours d'expériences issus du creusement d'ouvrages souterrains. Un exemple choisi ici, parmi d'autres, pour illustrer cette assertion, est le tunnel ferroviaire de Tartaiguille.

\section{- Les convergences du tunnel de Tartaiguille}

Le tunnel de Tartaiguille est un ouvrage de la ligne TGV Méditerranée, situé entre Valence et Montélimar, dont l'excavation a débuté en février 1996. Ce tunnel, d'une portée de $13 \mathrm{~m}$ (Fig. 2), d'une longueur de $2430 \mathrm{~m}$, traverse plusieurs faciès géologiques dont les marnes du Stampien dans sa partie centrale et le massif marneux de l'Aptien dans sa partie Sud (André et al., 1999). Dans ce dernier, l'excavation du tunnel a été réalisée en section divisée avec mise en place d'un soutènement léger constitué de boulons et de béton projeté.

La résistance en compression simple, estimée à partir d'essais mécaniques en cours de projet, variait entre $5 \mathrm{MPa}$ et $12 \mathrm{MPa}$ (Charmetton, 2001).

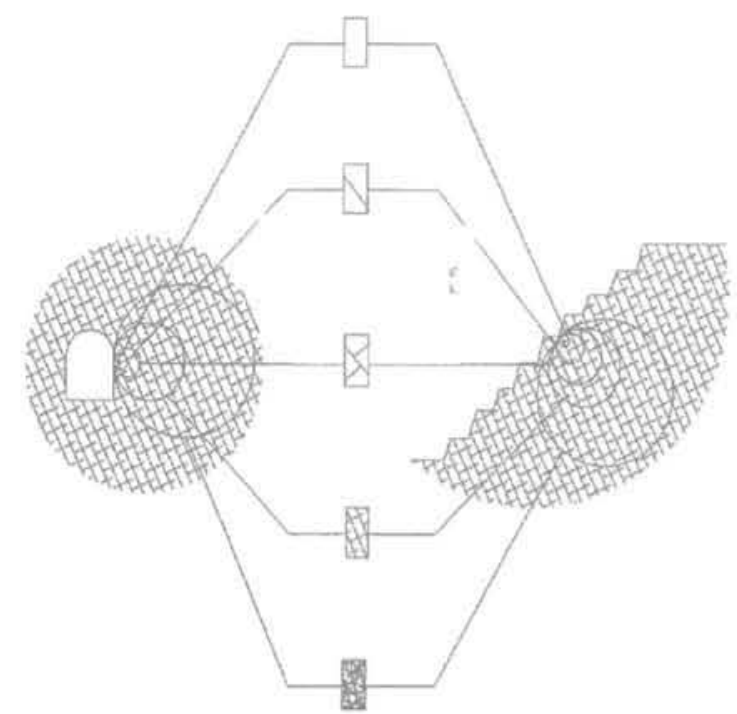

FO. 1 Illustration de l'effet d'échelle dans un massif fracturé.

The scale effect in a fractured rock mass.
L'état des contraintes initial est caractérisé par une contrainte maximale de compression horizontale, de l'ordre de 1,2 fois la contrainte verticale, supposée correspondre au poids des terres sus-jacentes. Autour d'une section située sous une couverture de $40 \mathrm{~m}$, au droit de laquelle des déplacements significatifs sont apparus, l'état des contraintes initial a été estimé à $\sigma_{\mathrm{v}}=1 \mathrm{MPa}$ et $\sigma_{\mathrm{H}}=1,2 \mathrm{MPa}$. En considérant un comportement élastique linéaire et une géométrie circulaire, la contrainte maximale orthoradiale en clé de voûte, en l'absence de soutènement, pouvait atteindre 2,6 MPa, soit une contrainte significativement plus faible que la valeur moyenne, voire minimale, de la résistance en compression simple mesurée en laboratoire.

Lors du creusement de l'ouvrage dans les marnes de l'Aptien, dès le creusement de la demi-section supérieure, des convergences relativement importantes sont apparues, sans signe de stabilisation à court terme. Ces mouvements se sont accentués lors du creusement de la demi-section inférieure, accompagnés d'une fissuration du soutènement en béton projeté.

Les convergences observées au niveau du raccordement rein/radier peuvent se justifier par le développement d'un mécanisme de cisaillement induit par la géométrie et le creusement en section divisée. Cette hypothèse semble confirmée par l'arrêt des convergences après un renforcement des piédroits. Il est par contre moins trivial de justifier, par les calculs, l'initiation et le développement de ce mécanisme de dégradation.

Des modélisations élastoplastiques (Laigle, 2004) ont été réalisées afin de comprendre le comportement observé et retrouver les convergences mesurées. Elles n'ont pas permis de retrouver les amplitudes des déplacements mais ont montré que, pour enclencher un mécanisme susceptible d'expliquer le comportement de l'ouvrage, il était nécessaire de retenir une résistance en compression simple de l'ordre de $1 \mathrm{MPa}$. II existe donc un biais entre la valeur de la résistance déduite du comportement de l'ouvrage et celle mesurée en laboratoire (Charmetton, 2001).

Cet exemple s'inscrit dans le cadre des roches tendres marneuses, pouvant être considérées comme ( continues $)$ à une échelle décamétrique autour des ouvrages. L'effet d'échelle, lié au taux de défauts structurels et de discontinuités matricielles, ne semble pas suffisant pour justifier la réduction des caractéristiques mécaniques in situ des marnes de Tartaiguille.

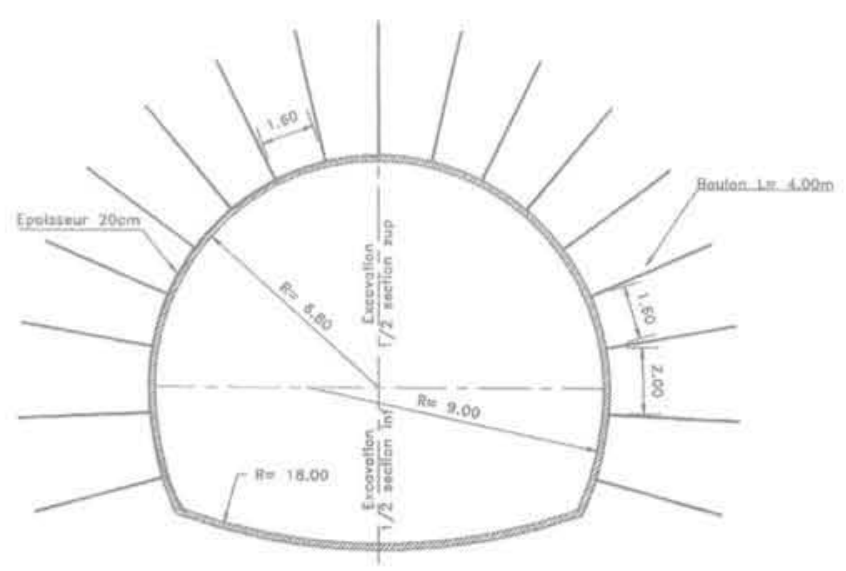

FG, 2 Section type du tunnel de Tartaiguille. Section of the Tartaiguille tunnel. 
Après l'énumération des principaux facteurs susceptibles d'expliquer la réduction de la résistance de la roche in situ, le comportement différé des roches est abordé via son identification en laboratoire et sur ouvrages et la notion de résistance à long terme qui lui est implicitement associée. Un modèle de comportement radoucissant intégrant l'effet du temps est proposé, suivi de ses applications à l'excavation d'une galerie circulaire.

\section{2}

\section{Problématique}

Dans le cas du tunnel de Tartaiguille et dans d'autres configurations relativement simples, les difficultés pour retrouver le comportement des ouvrages interpellent. Il paraît nécessaire de s'interroger sur les différents facteurs susceptibles de justifier cette inadéquation entre la prédiction et le comportement. observé. Sans préjuger de leur prééminence respective, certains facteurs d'influence peuvent être proposés : l'effet d'échelle, l'effet de structure, les conditions d'excavation, le chemin de contraintes, la présence d'un fluide au sein de la matrice et l'effet d'un couplage hydraulique-mécanique, les conditions de prélèvement, de remaniement et de conservation, en particulier la désaturation éventuelle des échantillons, et enfin le comportement différé du matériau.

\section{- L'effet d'échelle}

L'effet d'échelle est la notion qui vient généralement à l'esprit d'un mécanicien des roches lorsqu'il doit estimer la résistance d'un massif à une échelle d'analyse compatible avec les dimensions d'un ouvrage souterrain. Cet " effet d'échelle » conduit à réduire les caractéristiques mécaniques avec l'augmentation de la dimension d'étude. Il est d'autant plus prononcé que le milieu est fracturé et que le comportement global du massif est régi par les discontinuités préexistantes. A contrario, il n'a qu'un effet limité, et ne peut totalement justifier le cas particulier du tunnel de Tartaiguille, dans des marnes assimilées à un milieu homogène et continu à l'échelle de l'ouvrage.

\section{- L'effet de structure}

L'initiation et le développement d'un mécanisme de dégradation et de ruine éventuel d'un ouvrage ne sont pas uniquement liès à des données intrinsèques au matériau mais dépendent aussi des conditions de chargement et aux limites de la « structure $»$ : c'est ce qui peut être qualifié sous la terminologie « effet de forme » (Diederichs, 2002). Ainsi, l'initiation du mécanisme de ruine dépend, entre autres, de la géométrie de la cavité (changement de rayon de courbure des parois privilégiant l'initiation d'un mécanisme de cisaillement, longpans verticaux privilégiant le développement d'instabilité de forme...). Ceci sous-entend que la résistance mécanique, en particulier la résistance en compression, mesurée en laboratoire sur des échantillons cylindriques, n'est pas une donnée intrinsèque du matériau et ne correspond qu'à un chemin de contrainte particulier appliqué avec des conditions aux limites spécifiques.

\section{- Le chemin de contraintes}

Les calculs d'ouvrages souterrains linéaires sont généralement réalisés à partir de démarches d'analyse ou de modélisations uni ou bidimensionnelles. Par conséquent, le chemin des contraintes pris en compte dans ces approches est différent de celui induit en réalité par le creusement. Des études réalisées en configuration 3D par Kleine (2006) montrent que le passage du front de taille d'un tunnel circulaire s'accompagne d'un accroissement momentané du déviateur et d'un phénomène de rotation des contraintes. Cette augmentation est susceptible d'induire une dégradation physique et mécanique de la roche, modifiant le comportement ultérieur du massif rocheux en arrière du front.

\section{- Les conditions d'excavation}

Les modẻlisations n'intègrent généralement pas les conséquences immédiates et locales des méthodes de creusement et en particulier la dégradation pouvant être induite par l'usage d'explosifs ou d'outils d'excavation. Dans l'exemple de Tartaiguille, le processus de creusement ne semble pas pouvoir justifier le comportement observé.

\section{- Les couplages polyphasiques}

Les roches présentent une porosité, ouverte ou fermée, plus ou moins importante en fonction de leur nature géologique et de leur histoire, remplie de liquide et/ou de gaz. Des couplages existent entre le comportement macroscopique mécanique de la matrice rocheuse et les fluides présents dans ses vides. L'importance de ces couplages peut être quantifiée par différents facteurs comme, par exemple, le coefficient de Biot qui sera d'autant plus faible que la porosité sera réduite, ou encore la perméabilité qui dépendra elle aussi de cette porosité. A noter que ces caractéristiques évoluent lors d'un chargement mécanique, en fonction du taux de dégradation de la matrice rocheuse.

\section{- Prélèvement et remaniement}

Le carottage et la préparation des échantillons qui sont testés en laboratoire s'accompagnent:

- d'un endommagement et d'un échauffement éventuel de la roche qui entraîne une diminution des caractéristiques mécaniques:

- d'une désaturation immédiate (expansion de l'échantillon) ou différée (stockage et séchage) qui s'accompagne au départ d'un accroissement des propriétés mécaniques apparentes.

En apparence, ces deux mécanismes sont potentiellement susceptibles de s'annihiler. Cependant, étant de natures différentes, le comportement apparemment intrinsèque de la roche est fondamentalement modifié par ces phénomènes.

\section{- Le comportement différé}

La notion de comportement a différé n ou de comportement “visqueux » est associée à un effet du temps sur la réponse de la roche à une sollicitation mécanique, indépendamment des phènomènes décrits ci-dessus, et en particulier des couplages hydro-mécaniques.

A travers la notion de résistance à long terme qui lui est implicitement associẻe, il sera mis en évidence par la suite que le comportement visqueux est un phénomène susceptible de justifier significativement la décroissance de résistance apparente entre le laboratoire et l'ouvrage.

\section{3}

\section{Le comportement différé des roches}

\section{1}

\section{Mise en évidence}

En laboratoire, le comportement différé des roches est généralement mis en évidence à partir de trois 
types d'essai : des essais de fluage, des essais de relaxation et des essais de chargement mécanique à vitesse imposée.

L'existence du fluage dans les marnes de Tartaiguille a été mise en évidence par les mesures de convergences réalisées in situ et présentées sur la figure 3. Quatre sections sont considérées ici, chacune ayant été marquée par un arrêt de chantier d'une durée approximative de 25 jours. Durant la phase d'interruption du creusement, les convergences des parois latérales continuent à augmenter significativement.

Tous les géomatériaux, depuis les argiles, les argiles indurées, jusqu'aux roches cristallines raides, développent ce phénomène de comportement différé à l'échelle macroscopique, de manière plus ou moins significative, en fonction des conditions de contraintes, de température ou de la présence de fluides interstitiels. Ce phénomène est physiquement associé à des mécanismes différents selon la lithologie et la structure de l'espace poreux. Ce comportement est rarement quantifié au stade d'un projet d'ouvrage, soit parce qu'il n'a pas, a priori, été identifié comme ( significatif »), soit parce qu'il est difficile à quantifier à partir d'essais de laboratoire (cinétique trop lente).

Deux informations sont - ou se devraient d'être disponibles afin de quantifier partiellement ce comportement différé et de le prendre en compte implicitement ou explicitement dans la démarche d'analyse et de conception d'un ouvrage. Il s'agit de la résistance maximale en compression, voire le critère de résistance, à une vitesse de chargement de référence (généralement $10^{-5}-10^{-6} / \mathrm{s}$ ) et la résistance à long terme.

\section{Notion de résistance à long terme}

De par le comportement ( visqueux » que présentent les géomatériaux, la résistance devient dépendante du temps ou de la vitesse. Il convient donc de définir la notion de " résistance à long terme » qui correspond au seuil limite en contrainte en deçà duquel le comportement du matériau sous sollicitation mécanique se stabilise et n'évolue plus.

La définition et l'estimation de cette résistance dite " à long terme » ne font pas l'objet d'un consensus et restent sujettes à discussion.

Dans le domaine des faibles contraintes de confinement, et donc de comportement fragile, le seuil de résistance à long terme peut être assimilé soit :

- au seuil d'initiation de la fissuration et donc à l'initiation de la dilatance (entre $30 \%$ et $50 \%$ de la résistance en compression simple $\sigma$ ) (Aubertin, 2000) ;

- au seuil d'enclenchement d'une fissuration (cinstable ), correspondant au point d'inflexion de la courbe des déformations volumiques (entre $70 \%$ et $80 \%$ de $\sigma_{c}$ ) (Martin et Chandler, 1994).

Les travaux issus de l'analyse de Schmidtke et Lajtai (1985) ont permis de définir la résistance à long terme du granite du lac du Bonnet autour de $45 \%$ de $\sigma_{c}$. Les résultats obtenus par Wiid (1970) sur une dolerite montrent que le point d'initiation de la fissuration est une meilleure approximation de la résistance à long terme

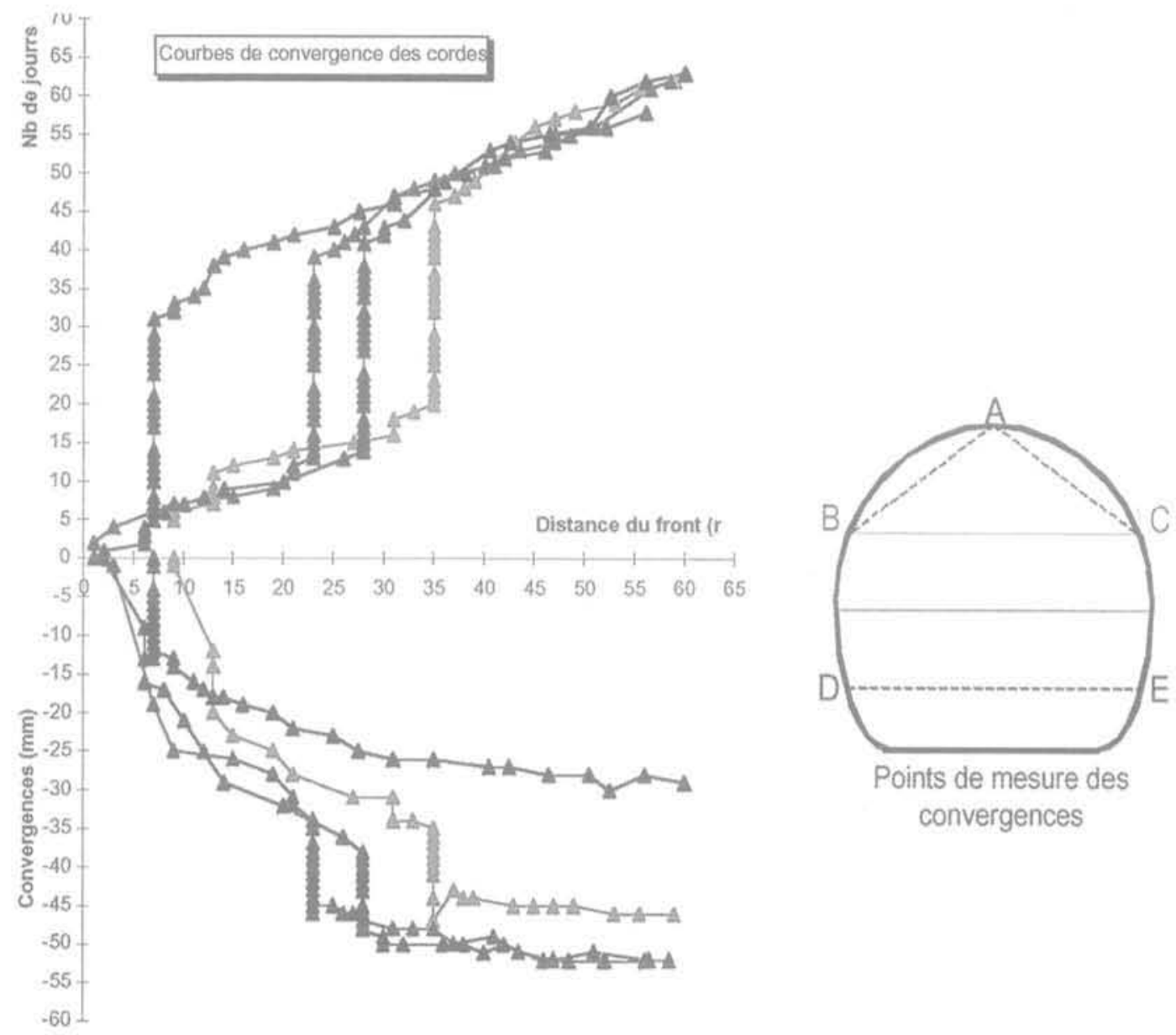

FIG.3 Mesures de convergences réalisées sur quatre sections du tunnel de Tartaiguille. Evolution of convergences around four sections of the Tartaiguille tunnel. 
que le point d'enclenchement de la fissuration instable. Dans ses études, Aubertin (2000) retient comme seuil de résistance à long terme le point d'initiation de la dilatance. Il associe la seconde définition à des périodes de temps plus courtes (jours, semaines) (Aubertin et Simon, 1997). Par ailleurs, la seconde définition, relativement subjective, contredit la nature " intrinsèque $»$ - avancée par Martín et Chandler (1994) - de ce seuil, indépendant de la géométrie, des conditions aux limites de chargement et surtout de la vitesse de sollicitation. Ces différents retours d'expérience conduisent donc à retenir la première définition.

Par ailleurs, d'après Lau et Chandler (2004), ces deux définitions sous-estiment la résistance à long terme de la roche sous de plus fortes contraintes de confinement. La question reste donc ouverte sur la définition du critère de résistance à long terme sur une plus grande gamme de contraintes.

D'après les résultats expérimentaux synthétisés par Cho et Christiansson (2002), un seuil de résistance insitu, différent de celui estimé en laboratoire, a été défini et approché par l'équation $\frac{\sigma_{\max }}{\sigma_{\min }}=20$. Une analyse « micro » (Kleine, 2006) conduirait à assimiler cette limite au seuil de résistance à long terme, pour des contraintes de confinement élevées.

Les hypothèses avancées ici sont schématiquement illustrées sur la figure 4. Elles s'appuient sur une conceptualisation simple du comportement mécanique d'une roche sous un chargement de compression. Elles sont intégrées dans un modèle rhéologique élastovisco-plastique, développé en collaboration par EDF et le LaEGO. Cette loi de comportement, décrite et appliquée par la suite, a été validée par la simulation du comportement de plusieurs ouvrages, dont le tunnel de Tartaiguille.

\section{Application aux ouvrages souterrains}

Le creusement d'un tunnel est un processus conduisant à charger le terrain de manière relativement lente. Pour un tunnel circulaire de $10 \mathrm{~m}$ de diamètre, creusé à une vitesse moyenne de $10 \mathrm{~m} / \mathrm{j}$, la vitesse de déformation est de l'ordre de $10^{-9} / \mathrm{s}$, soit 1000 à 10000 plus faible que celle appliquée en laboratoire. Comme le montre la figure 5 qui a été établie par une analyse en retour sur les marnes de l'Aptien, la diminution de la vitesse de déformation se traduit par une réduction significative de la résistance. Ainsi, pour une configuration habituellement considérée comme du « court terme ») et ne faisant donc pas a priori intervenir le comportement différé du matériau, la résistance du massif mobilisée durant le creusement de l'ouvrage est beaucoup plus faible que celle définie par les essais de laboratoire.

Une vitesse de déformation in situ de l'ordre de $10^{-9 / 5}$ conduit à une résistance du massif de l'ordre de $1 \mathrm{MPa}$, soit une diminution de l'ordre de $80 \%$ par rapport à la résistance mesurée en laboratoire.

Cette analyse montre que l'existence d'un comportement différé de la roche, aussi faible soit-ill, s'accompagne d'une réduction à relativement court terme de la résistance apparente de la roche. Ce phénomène est susceptible de justifier des observations sur certains ouvrages difficilement explicables par la seule notion d'effet d'échelle. En conséquence, le comportement différé des roches est un phénomène important à prendre en compte dans certaines configurations, soit par la mise en ceuvre de modèles viscoplastiques pertinents, soit par des approches plus simples ne reproduisant pas le comportement différé mais considérant un seuil de résistance maximale représentatif.

\section{4}

\section{Modèle rhéologique de comportement}

Les modélisations présentées par la suite ont été réalisées en mettant en œuvre un modèle de comportement viscoplastique, reprenant les notions décrites et discutées précédemment. Il s'agit d'un modèle rhéologique, appelé modèle $L \& K$, initialement élastoplastique, étendu à une version viscoplastique (Kleine, 2006),

\section{1}

\section{Notations et partition du tenseur des déformations}

Soient $\mathrm{g}$ le tenseur des contraintes, s le tenseur déviatoire, $\bar{I}_{1}$ la trace de $g$, et $s_{\| 1}$ le second invariant de $\underline{\underline{s}}$ :

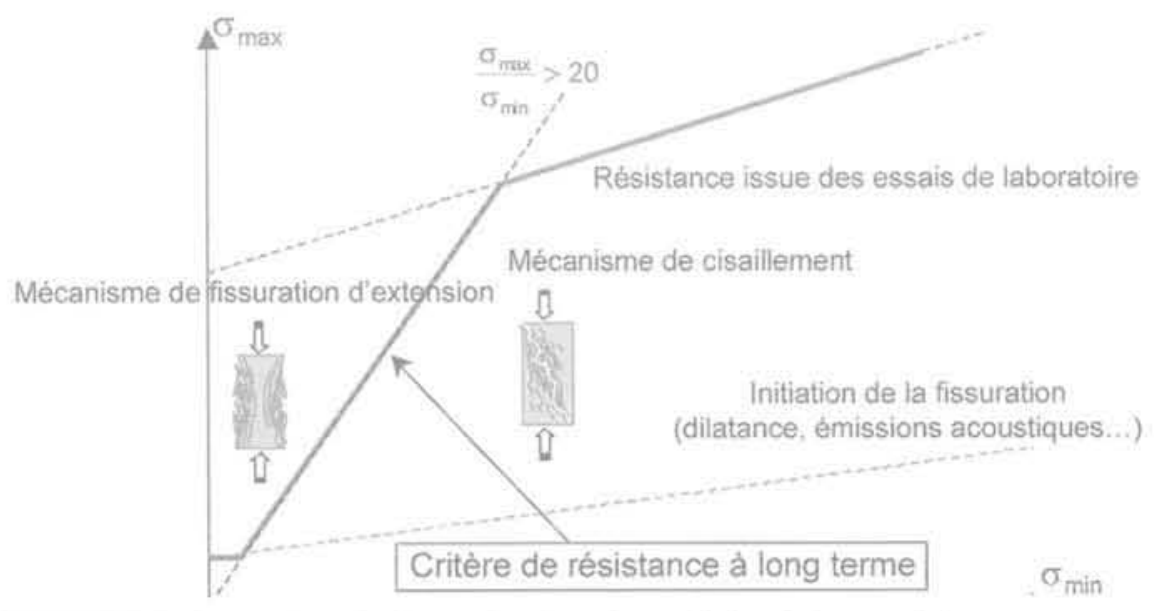

FiG. 4 Principe de détermination du seuil de résistance à long terme. Definition of the long-term strength threshold. 


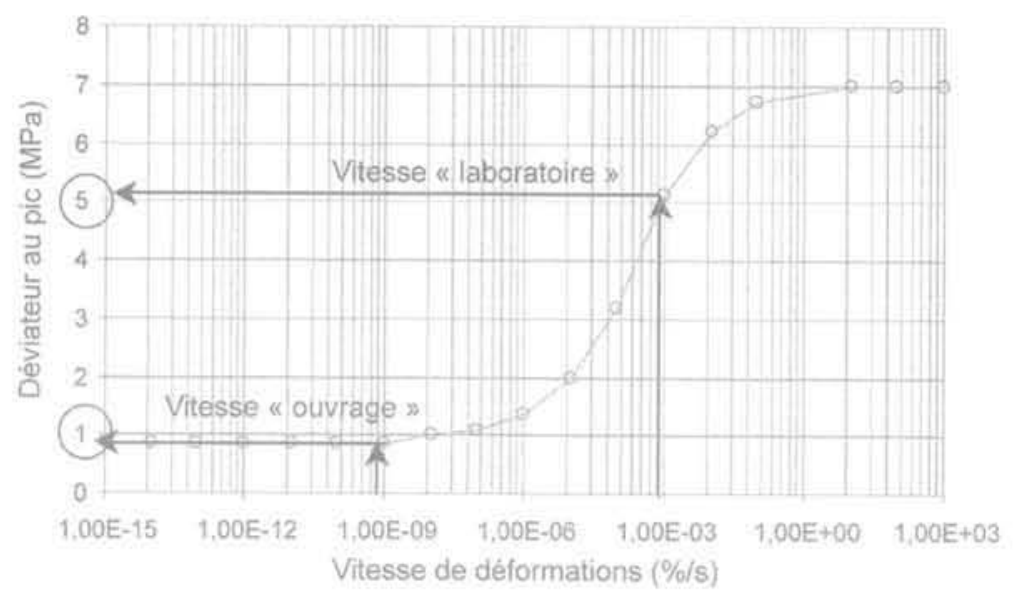

FiG.5 Exemple d'évolution de la résistance en compression simple avec la vitesse de déformation. Evolution of UCS with strain rate.

$S_{i j}=\sigma_{i j}-\frac{\operatorname{tr}\left(\sigma_{i j}\right)}{3} \delta_{i j} \quad I_{1}=\operatorname{tr}\left(\sigma_{i j}\right) \quad S_{I I}=\sqrt{S_{i j} \cdot S_{j}}$. L'angle de

Lode est défini par : $\theta=\frac{1}{3} \arccos \left(2^{1 / 2} 3^{3 / 2} \frac{\operatorname{det}\left(\sigma_{0}\right)}{\mathrm{s}_{11}{ }^{3}}\right)$

Soient $\underline{\underline{\varepsilon}}, \underline{\underline{\varepsilon}} \underline{\varepsilon}^{c}, \underline{\varepsilon}^{i}$, les tenseurs des déformations totales,

élastiques et irréversibles respectivement, $\varepsilon_{v} \varepsilon_{v}^{e}$, $\varepsilon_{v}^{i}$, les déformations volumiques totales, élastiques et irréversibles respectivement $e_{0}$ la partie déviatoire de $\varepsilon_{i 11}$ et $\gamma^{\prime}$ la distorsion irréversible:

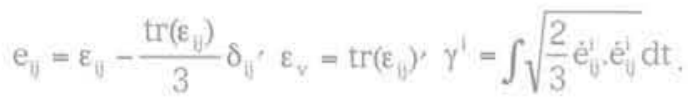

Conformément à la théorie de l'élastoplasticité :

$$
\underline{\underline{\varepsilon}}=\underline{\underline{\varepsilon}}^{e}+\underline{\underline{\varepsilon}}^{\prime}
$$

La déformation irréversible se décompose en un terme de déformation irréversible instantanée $\varepsilon^{\text {ep }}$ et un terme de déformation irréversible différée $\varepsilon^{v p}$. Chacune de ces composantes est respectivement générée par un mécanisme élastoplastique et un mécanisme viscoplastique :

$$
\underline{\underline{\varepsilon}}^{\underline{\underline{\varepsilon}}}=\underline{\underline{\varepsilon}}^{\text {ep }}+\underline{\underline{\varepsilon}}^{\text {vp }}
$$

\section{2}

\section{Mécanisme élastique}

Les paramètres régissant le comportement élastique sont les modules de cisaillement $\mathrm{G}$ et volumique $\mathrm{K}$. La loi choisie est de type hypoélastique, de forme générale :

$$
\dot{\varepsilon}_{i j}^{e}=\frac{1}{2 G} \dot{S}_{i j}+\frac{1}{9 K} I_{3} \delta_{i j}
$$

Les modules $\mathrm{K}$ et $\mathrm{G}$ dépendent de l'état des contraintes

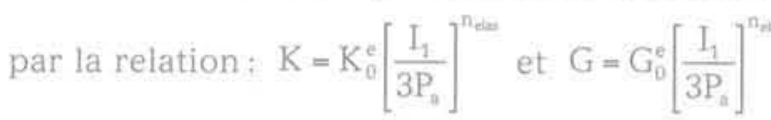

$\mathrm{P}_{\mathrm{n}}$ désigne la pression atmosphérique, généralement prise égale à $100 \mathrm{kPa}$ et $\mathrm{K}_{0}^{\mathrm{e}}, \mathrm{G}_{0}^{\mathrm{e}}$ et $\mathrm{n}_{\text {cias }}$ sont des paramètres du modèle définis à partir d'essais de laboratoire.

\section{3}

\section{Mécanismes élastoplastiques}

La surface de charge élastoplastique, écrite dans un formalisme en invariants, est définie par les équations suivantes :

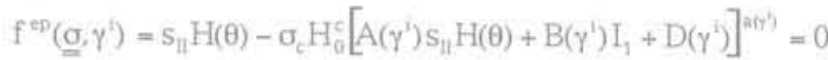

$$
\begin{aligned}
& \mathrm{A}\left(\gamma^{j}\right)=-\frac{\mathrm{m}\left(\gamma^{\prime}\right) \mathrm{k}\left(\gamma^{i}\right)}{\sqrt{6} \sigma_{c} h_{0}^{c}} \quad B\left(\gamma^{\prime}\right)=\frac{m\left(\gamma^{j}\right) \mathrm{k}\left(\gamma^{\prime}\right)}{3 \sigma_{\mathrm{c}}} \quad \mathrm{D}\left(\gamma^{\prime}\right)=s\left(\gamma^{i}\right) \mathrm{k}\left(\gamma^{i}\right) \\
& k\left(\gamma^{1}\right)=\left(\frac{2}{3}\right)^{\overline{2 a\left(y^{\prime}\right)}}
\end{aligned}
$$

$h(\theta)=(1-\gamma \cos 3 \theta)^{1 / 6} h_{0}^{\epsilon}=h\left(0^{\circ}\right)=(1-\gamma)^{2 / 6} h_{0}^{c}=h\left(60^{\circ}\right)=(1+\gamma)^{1 / 6}$ $\mathrm{H}(\theta)=\frac{\mathrm{H}_{0}^{c}+\mathrm{H}_{0}^{e}}{2}+\left(\frac{\mathrm{H}_{0}^{\mathrm{c}}-\mathrm{H}_{0}^{\mathrm{c}}}{2}\right)\left(\frac{2 \mathrm{~h}(\theta)-\left(\mathrm{h}_{0}^{\mathrm{c}}+\mathrm{h}_{0}^{\mathrm{c}}\right)}{\mathrm{h}_{0}^{\mathrm{c}}-\mathrm{h}_{0}^{\mathrm{c}}}\right)$

$\sigma_{\mathrm{c}}$ désigne la résistance en compression simple, $\mathrm{H}_{0}^{c}$ est usuellement pris égal à hc et $\mathrm{H}_{0}^{\mathrm{e}}$ est un paramètre du modèle pilotant la résistance en extension. Sur un chemin de compression triaxiale $\left(\theta=0^{\circ}, \mathrm{s}_{\mathrm{II}}=\sqrt{\frac{2}{3}}\left(\sigma_{1}-\sigma_{3}\right), \mathrm{I}_{1}=\right.$, $\sigma_{1}+2 \sigma_{3}$ ) la surface de plasticité correspond à un critère de Hoek et Brown généralisé.

La surface de charge peut être visualisée dans le plan $(\pi)$ normal à l'axe hydrostatique $\mathrm{I}_{1}$ et en perspective dans l'espace des contraintes sur la figure 6 .

Les paramètres $m\left(\gamma^{\prime}\right)$, $s\left(\gamma^{\prime}\right)$ et a $\left(\gamma^{\prime}\right)$ évoluent selon des lois d'écrouissage spécifiques, traduisant la dégradation progressive des caractéristiques mécaniques de la roche à l'échelle macroscopique. Sous l'effet d'un chargement mécanique de compression, cet écrouissage induit un comportement non linéaire avant le pic de résistance (écrouissage positif) et un comportement post-pic radoucissant (écrouissage négatif).

Les déformations irréversibles instantanẻes sont obtenues à partir de la règle de normalité appliquée à une fonction potentielle $\mathrm{G}$, dont l'expression est donnée par (8).

Le mécanisme élastoplastique est virtuellement subdivisé en deux mécanismes (Fig. 7) :

- un mécanisme pré-pic, moteur de l'initiation d'une fissuration d'extension instantanée ; 


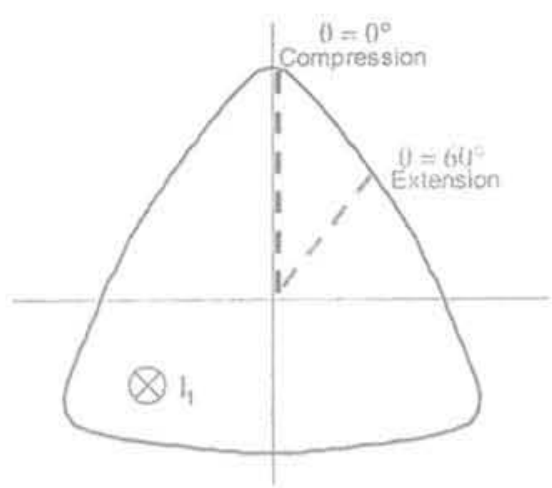

(a)

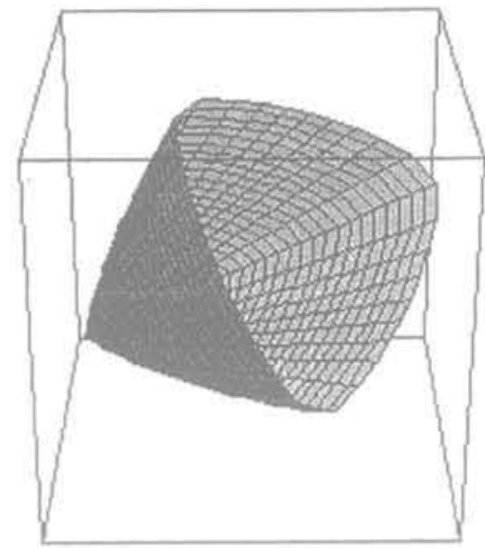

(b)

FG.6 Surface de charge dans le plan $\pi$ (a) et dans l'espace des contraintes (b), Yield surface in $\pi$-plane (a) and in stresses space (b).

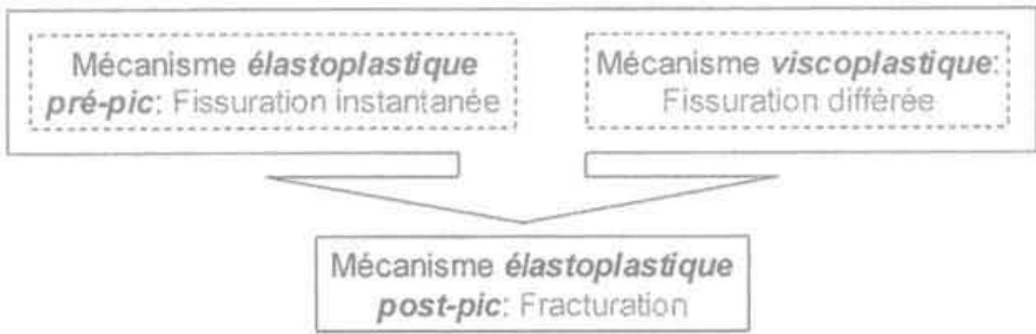

FG. 7 Conceptualisation des mécanismes du modèle $L \& K$. $L \& K$ constitutive model mecanisms.

- un mécanisme post-pic, traduisant la dégradation progressive du matériau, conséquence de l'initiation de la fissuration induite par les mécanismes pré-pic (instantanée) et viscoplastique (différée).

\section{4}

\section{Mécanisme viscoplastique}

Le calcul des déformations irréversibles différées $\varepsilon^{\mathrm{vp}}$ repose sur la théorie de Perzyna (1966). La vitesse de déformation viscoplastique s'exprime en fonction de la distance $F$ entre le point de charge et la surface viscoplastique (surcontrainte) :

$$
\underline{\underline{\varepsilon}}^{\mathrm{vp}}=\langle\Phi(\mathrm{F})\rangle \mathrm{G}
$$

La distance $F$ est quantifiée par une expression du type (5). G et $\Phi(F)$ caractérisent respectivement la direction et l'amplitude de la vitesse des déformations irréversibles. G s'obtient à l'aide des équations $(8)$ et $\Phi(F)$ est calculée par :

$$
\Phi(F)=A_{v}\left(\frac{F}{F_{0}}\right)^{n_{v}}
$$

$A_{v}$ et $n_{v}$ sont des paramètres du modèle et $F_{0}$ désigne la pression atmosphérique habituellement prise à $100 \mathrm{kPa}$.

\section{5}

\section{Comportement volumique}

La loi d'écoulement des mécanismes irréversibles est non associée. Si f désigne fep pour les mécanismes élastoplastiques et $\mathrm{F}$ pour le mécanisme viscoplastique, la fonction potentielle $\mathrm{G}$ est de la forme :
$\mathrm{G}=\frac{\partial \mathrm{f}}{\partial \dot{\delta}}-\left(\frac{\partial \mathrm{f}}{\partial \dot{o}^{\prime}} \mathrm{n}\right) \mathrm{n}$

avec $\mathrm{n}=\frac{\beta \frac{\mathrm{s}}{\mathrm{s}_{11}}-\mathrm{Id}}{\sqrt{\beta^{2}+3}}, \beta=\frac{\sqrt{6} \mathrm{~g}_{v}}{3-\mathrm{g}_{v}}, g_{v}=-\frac{2 \sin \psi}{1-\sin \psi}$

$\psi$ désigne l'angle de dilatance et sa détermination diffère selon le mécanisme.

Sur la base de justifications micromécaniques (Kleine, 2006), une surface caractéristique a été introduite, délimitant l'espace des contraintes en un domaine contractant et un domaine dilatant. La figure 8 illustre cette notion, dans le plan $\left(\sigma_{\min } ; \sigma_{\max }\right)$.

- Pour les mécanismes élastoplastique pré-pic et viscoplastique, $\psi=\psi_{0 \text { v }}$ est défini par :

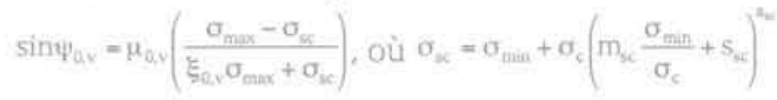

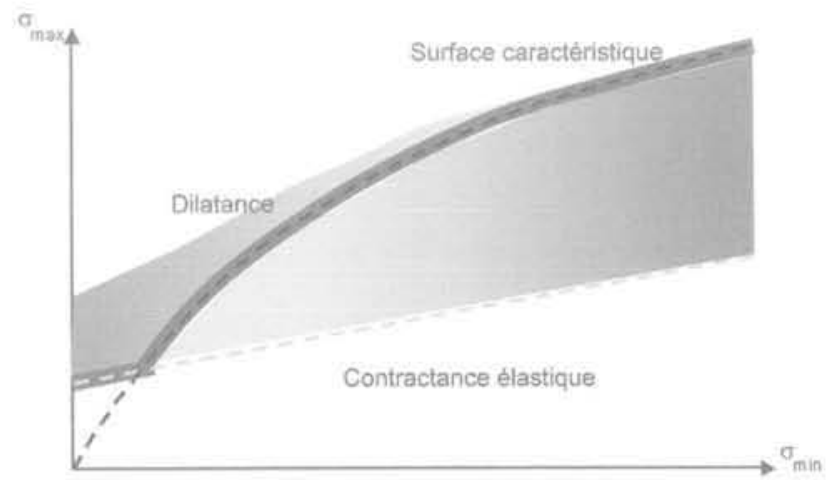

FG.8 Surface caractéristique et comportement volumique

Caracteristic threshold and volumetric behavior. 
$\mathrm{m}_{\mathrm{sc}}, \mathrm{s}_{\mathrm{sc}}$ et $\mathrm{a}_{\mathrm{sc}}$ désignent les valeurs des paramètres définissant la surface caractéristique et $\mu_{0, \mathrm{v}}$ et $\xi_{\text {i.v }}$ sont des paramètres strictement positifs du modèle.

Si le point de charge est en dessous de la surface caractéristique $\left(\sigma_{\max } \leq \sigma_{s c}\right)$, le comportement volumique est contractant ( $\sin \psi_{0, v} \leq 0$ ) tandis que si le point de charge est au-dessus de la surface caractéristique $\left(\sigma_{\max }>\sigma_{s c}\right)$, le comportement volumique est dilatant $\left(\sin \psi_{0, v}>0\right)$.

- Pour le mécanisme élastoplastique post-pic, $\psi=\psi_{1}$ )est calculé à partir des expressions suivantes

$\sin \psi_{1}=\mu_{1}\left(\frac{\alpha-\alpha_{\mathrm{max}}}{\xi_{1} \alpha-\alpha_{\mathrm{man}}}\right), \alpha=\frac{\sigma_{\max }+\tilde{\sigma}}{\sigma_{\min }+\tilde{\sigma}}, \alpha_{\mathrm{res}}=\left(\frac{\sigma_{\operatorname{mix}}}{\sigma_{\min }}\right)_{\mathrm{res}}=1+m_{\mathrm{min}}$

$\mathrm{m}_{\text {uit }}$, et $\mu_{1}$ et $\xi_{1}$ sont des paramètres du modèle.

Durant la phase de radoucissement post-pic, le comportement volumique est toujours dilatant, sauf à l'état résiduel où l'angle de dilatance s'annule (comportement purement frottant).

La quantité õ traduit l'effet de la cohésion sur la dilatance en l'assimilant à un confinement supplémentaire.

Si $\xi=1, \kappa=1$ et si la résistance en compression simple est nulle (cas d'un milieu pulvérulent), l'expression [10] correspond à la relation contrainte-dilatance de Rowe (1962).

\section{6}

\section{Couplage des mécanismes}

L'amplitude des déformations irréversibles instantanées et différées est fonction des positions respectives de la surface de charge élastoplastique fep et de la fonction de seuil viscoplastique $F(5)$. Ces surfaces sont supposées évoluer en fonction d'une seule variable d'écrouissage qui correspond à la déformation déviatoire plastique cumulée $\gamma$.

La distinction des mécanismes élastoplastiques préet post-pic ainsi que le couplage avec le mécanisme viscoplastique nécessitent une partition de la variable d'écrouissage $\gamma$. Si $\dot{\gamma}_{0}^{\prime}, \dot{\gamma}_{1}^{\prime}$ et $\dot{\gamma}_{\text {viss }}^{l}$ désignent respectivement les incréments de déformations irréversibles générées par les mécanismes pré-pic, post-pic et viscoplastique, leur contribution peut ou non intervenir dans la variable d'écrouissage relative à chaque mécanisme. Plus précisément:

- l'écrouissage positif du mécanisme élastoplastique pré-pic est fonction uniquement des déformations déviatoires plastiques pré-pic : $\dot{\gamma}_{0}$;

- le mécanisme viscoplastique évolue uniquement en fonction des déformations irréversibles différées : $\dot{\gamma}_{\mathrm{visc}}^{i}$; - l'écrouissage du mécanisme élastoplastique post-pic est un peu plus subtil. Physiquement, cette phase d'écrouissage négatif doit traduire la dégradation des propriétés mécaniques de la roche, proportionnellement à la dilatance. Mathématiquement, cela se traduit en faisant évoluer ou non le mécanisme post-pic :

Si le point de charge n'a pas encore atteint la surface caractéristique (Fig. 8), le mécanisme post-pic n'est pas activé : $\gamma=0$. Dès que le point de charge dépasse la surface caractéristique, le mécanisme post-pic s'enclenche et évolue en fonction de :

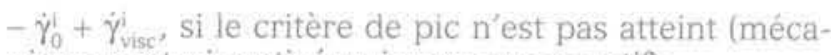
nisme post-pic activé mais pas encore actif) ;

$-\dot{\gamma}_{1}+\dot{\gamma}_{\text {vise }}$, en phase de radoucissement post-pic.
Ces conditions génèrent un couplage fondamental entre les trois mécanismes d'évolution du matériau. L'activation du mécanisme viscoplastique induit non seulement des déformations différées, mais génère implicitement un écrouissage négatif de la surface de charge du mécanisme élastoplastique post-pic. Ce couplage permet de traduire une dépendance avec le temps des caractéristiques mécaniques maximales de la roche, et donc une dégradation progressive, sous l'effet d'un chargement mécanique variable ou constant, de la résistance de la roche.

\section{7}

\section{Intégration numérique et critères d'interprétation}

Le modèle $L \& K$ a été intégré, en langage $C_{++}$, dans les logiciels FLAC et FLAC ${ }^{3 D}$. Il a été développé dans le cadre de projets d'ingénierie et doit permettre une interprétation rapide, claire et pertinente de certains critères de dimensionnement. Quelques critères sont cités ci-après, mais seuls les deux premiers seront exploités par la suite.

- Niveau de dégradation de la roche

La formulation du modèle est telle qu'elle permet d'affecter, à un niveau d'écrouissage donné, un état physique de la matrice rocheuse. Ceci permet d'envisager une interprétation qualitative des résultats de calcul et ainsi de visualiser aisément le mécanisme de comportement de l'ouvrage. La roche peut être dans cinq états :

- état 0 : roche à l'état initial, sans fissuration néoformée : - état 1 : roche fissurée pré-pic :

- état 2 : roche fissurée post-pic :

- état 3 : roche fracturée, ayant perdu toute cohésion à l'échelle « macroscopique » ;

- état 4 : roche à l'état résiduel, présentant un comportement purement frottant.

\section{- Index de fissuration}

Un indicateur du degré de fissuration, dit « index de fissuration 1 , a été introduit afin de traduire la perte de résistance du matériau. Il est noté $\mathrm{D}$ et varie entre 0 et 1 : $-D=0$ si le matériau est intact, s'il présente encore toute sa résistance $\alpha$ intrinsèque $)$,

$-D=1$ si le matériau a perdu toute sa résistance, toute sa cohésion.

\section{- Zone de fissuration d'extension}

Elle caractérise le domaine au sein duquel le matériau présente un comportement volumique dilatant. - Évaluation de l'accroissement de la perméabilité

Compte tenu du contexte des applications du modèle (stockage des déchets radioactifs), il est possible d'évaluer l'accroissement local de la perméabilité,

\section{5}

\section{Exemples}

Afin d'illustrer l'influence de la viscosité sur le comportement à " court et long termes » d'un tunnel, des modélisations élastoplastiques et viscoplastiques sont présentées sur une configuration axisymétrique. L'ouvrage est une galerie circulaire de rayon $R=4 \mathrm{~m}$ 


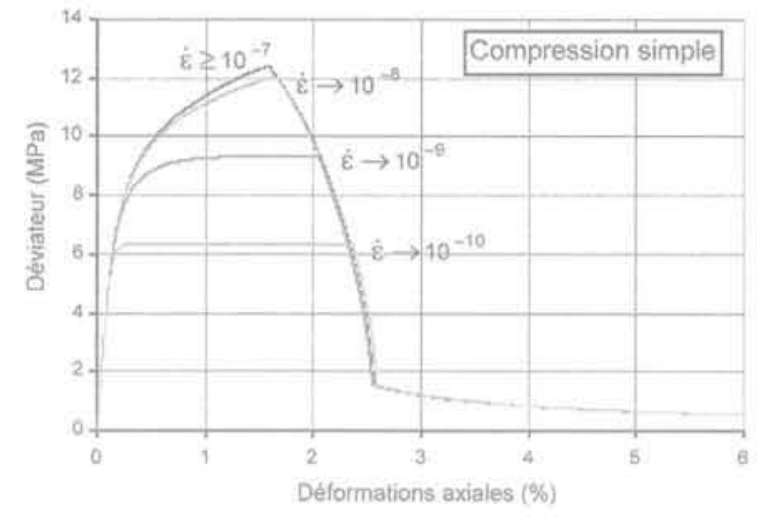

a) Essais triaxaux à vitesse imposée

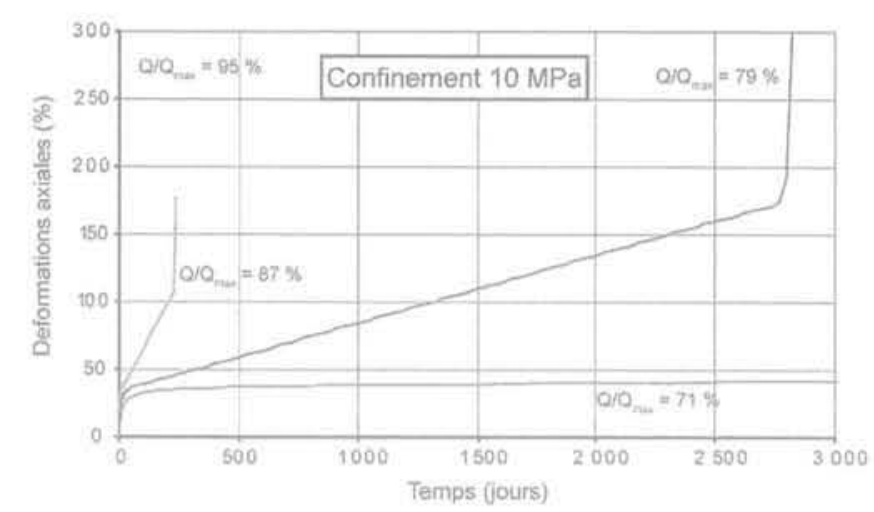

b) Essais de fluage

FIG. 9 Simulations d'essais triaxiaux. Simulations of laboratory tests.

et l'état des contraintes initial est supposé isotrope d'amplitude $\sigma_{0}=16,0 \mathrm{MPa}$. L'influence de plusieurs paramètres relatifs au scénario de construction est analysée, en terme de dégradation du massif :

- influence de la vitesse moyenne de creusement ;

- effet de la mise en œuvre d'un revêtement ;

- notion de stabilité à long terme.

5.1

\section{Identification des paramètres}

Le matériau considéré est de type « roche argileuse ») (Kleine, 2006). La figure 9 présente les simulations d'essais triaxiaux à différentes vitesses de chargement et des essais de fluage, pour plusieurs déviateurs de contraintes.

La cinétique de fluage retenue pour ce jeu de paramètres est très faible, à la limite des possibilités des appareils de mesure. Ce choix permet de se placer dans une configuration où le phénomène de comportement différé pourrait être considéré comme négligeable.

\section{9}

\section{Influence de la viscosité}

Une première comparaison consiste à visualiser les résultats de simulations en considérant simultanément les versions élastoplastique $\left(\mathrm{A}_{\mathrm{y}}=0\right.$ dans $\left.(7)\right)$ et viscoplastigue du modèle rhéologique. Sur un chemin triaxial de compression, simulé à une vitesse de $10^{-5} / \mathrm{s}$, les courbes contrainte/déformation obtenues avec les deux versions coincident. Le creusement de l'ouvrage est réalisé, de manière continue, à une vitesse de $2 \mathrm{~m} / \mathrm{j}$, sans mise en place de soutènement ni de revêtement.

La figure 11 montre une comparaison de l'état de dégradation de la roche autour du tunnel, entre les deux hypothèses de calcul retenues. Alors que le processus de creusement est généralement considéré comme du " court terme ", l'approche viscoplastique prédit une fracturation plus importante et évolutive en parois de l'ouvrage.

La figure 10 présente l'évolution de l'index de fissuration en fonction de la distance à la paroi (normalisée par le rayon). Pour rappel, la roche est fracturée sí l'index de fissuration vaut 1. D'après le graphique,

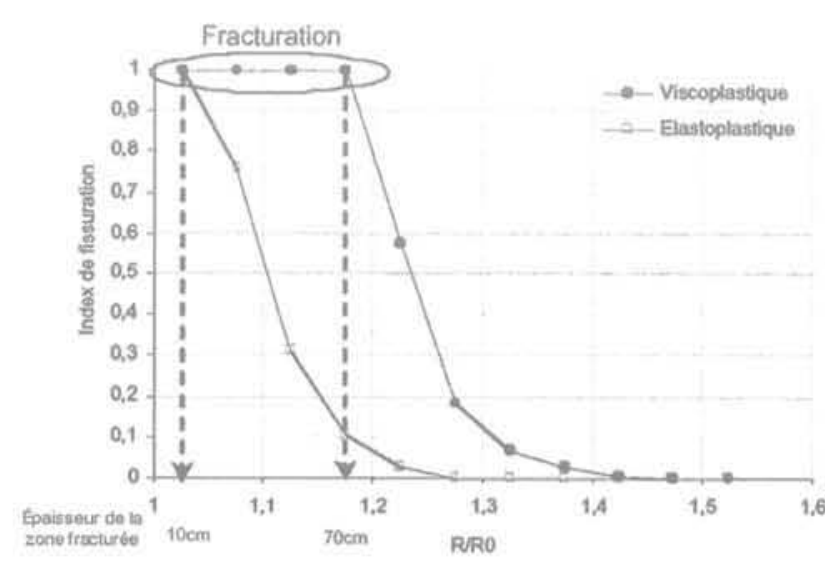

FIG. 10 Influence du modèle de comportement sur l'étendue de la zone fracturée. Influence of viscosity on fractured zone thickness

l'épaisseur de la roche fracturée, 18 jours après le passage du front, est estimée autour de $17,5 \% \mathrm{R}$, avec I'approche viscoplastique, et autour de $2,5 \% \mathrm{R}$, avec l'approche élastoplastique,

Tandis que ces deux modèles coïncident sur des essais de compression triaxiale réalisés à des vitesses de chargement de $10^{-5} / \mathrm{s}$ (Fig. 9a), l'extrapolation à l'ouvrage, avec des conditions spécifiques de chargement, montre des comportements différents en fonction de la prise en compte ou non de la viscosité du massif.

\section{3}

\section{Influence de la vitesse de creusement}

Des simulations du creusement du tunnel ont été réalisées en considérant plusieurs vitesses d'avancement du chantier. Les calculs sont réalisés avec le modèle viscoplastique. L'excavation est faite de manière continue, sans interruption. La figure 12 montre l'influence de la vitesse de creusement sur l'extension de la zone fracturée, via l'évolution de l'index de fissuration. Selon la vitesse d'excavation, l'épaisseur de la zone fracturée, prédite par le calcul, varie de $12,5 \% \mathrm{R}$ pour un creusement excessivement rapide, à $17,5 \%$ R pour un creusement très lent. 
Approche élastoplastique
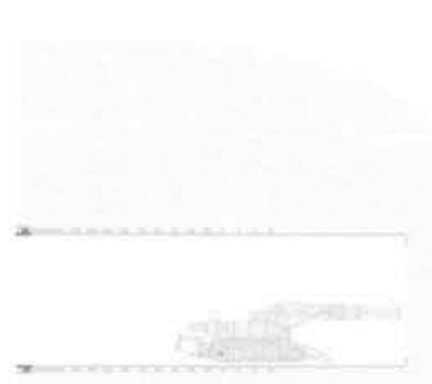

Fissưré pré-pic
Approche viscoplastique

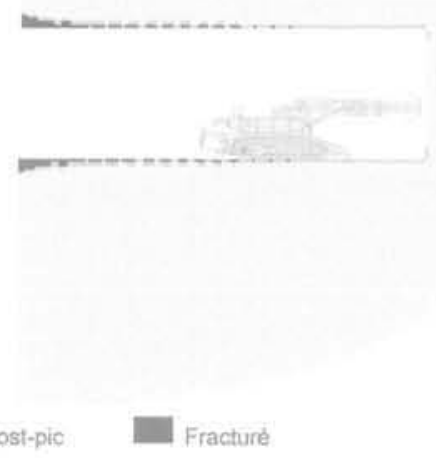

FiG. 11 Influence du modèle de comportement sur la dégradation du massif en cours de creusement.

Effect of the viscosity on the rock mass degradation.

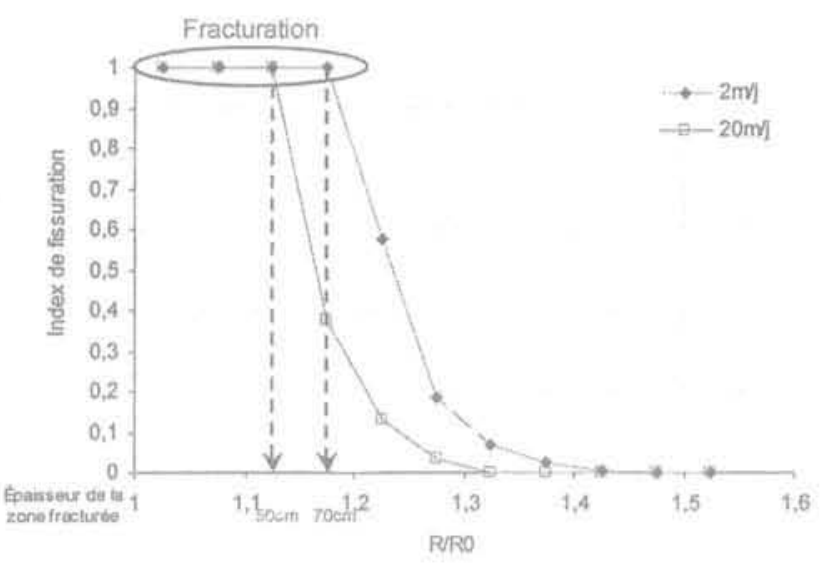

FG. 12 Influence de la vitesse de creusement sur T'extension de la zone fracturée

Influence of excavation rate on fractured zone thickness.

Une réduction de la vitesse moyenne d'excavation s'accompagne d'une dégradation supplémentaire du massif, avec comme corollaire un accroissement du risque d'instabilité de l'ouvrage en l'absence provisoire d'un soutènement ou revêtement.

\section{4}

\section{Influence d'un revêtement}

Avec les hypothèses de calculs retenues, le nombre de stabilité de l'ouvrage est estimé autour de 1.6, ce qui caractérise un ouvrage relativement aisé à concevoir en terme de soutènement et de revêtement. Les modélisations présentées ci-dessus montrent qu'effectivement, à l'échelle de quelques jours, la stabilité générale de l'ouvrage n'est pas critique. Toutefois, les conditions de symétrie du calcul et l'absence de gravité sont suscep-

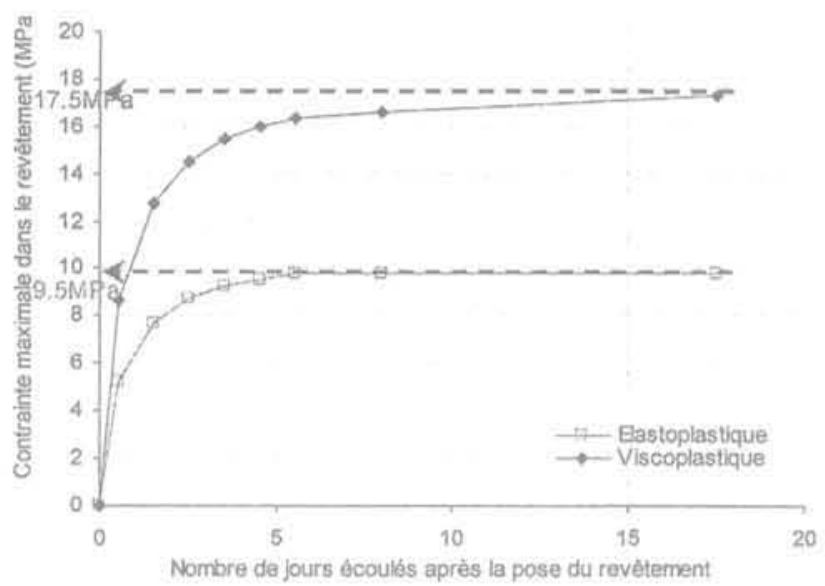

FIG. 13 Évolution des efforts dans le revêtement pendant la phase d'excavation.

Stresses evolution in shotcrete during the digging process.

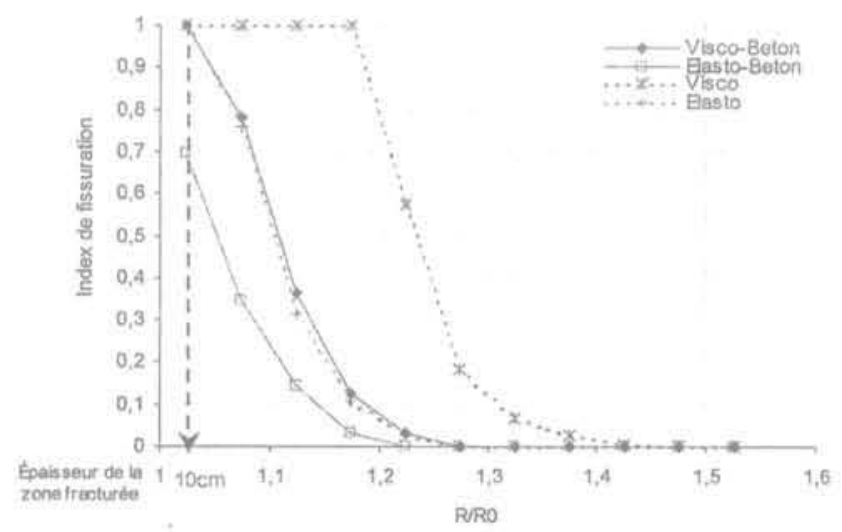

FiG. 14 Effet du revêtement sur le développement de la fissuration. Effects of the support on fissuration growth. 


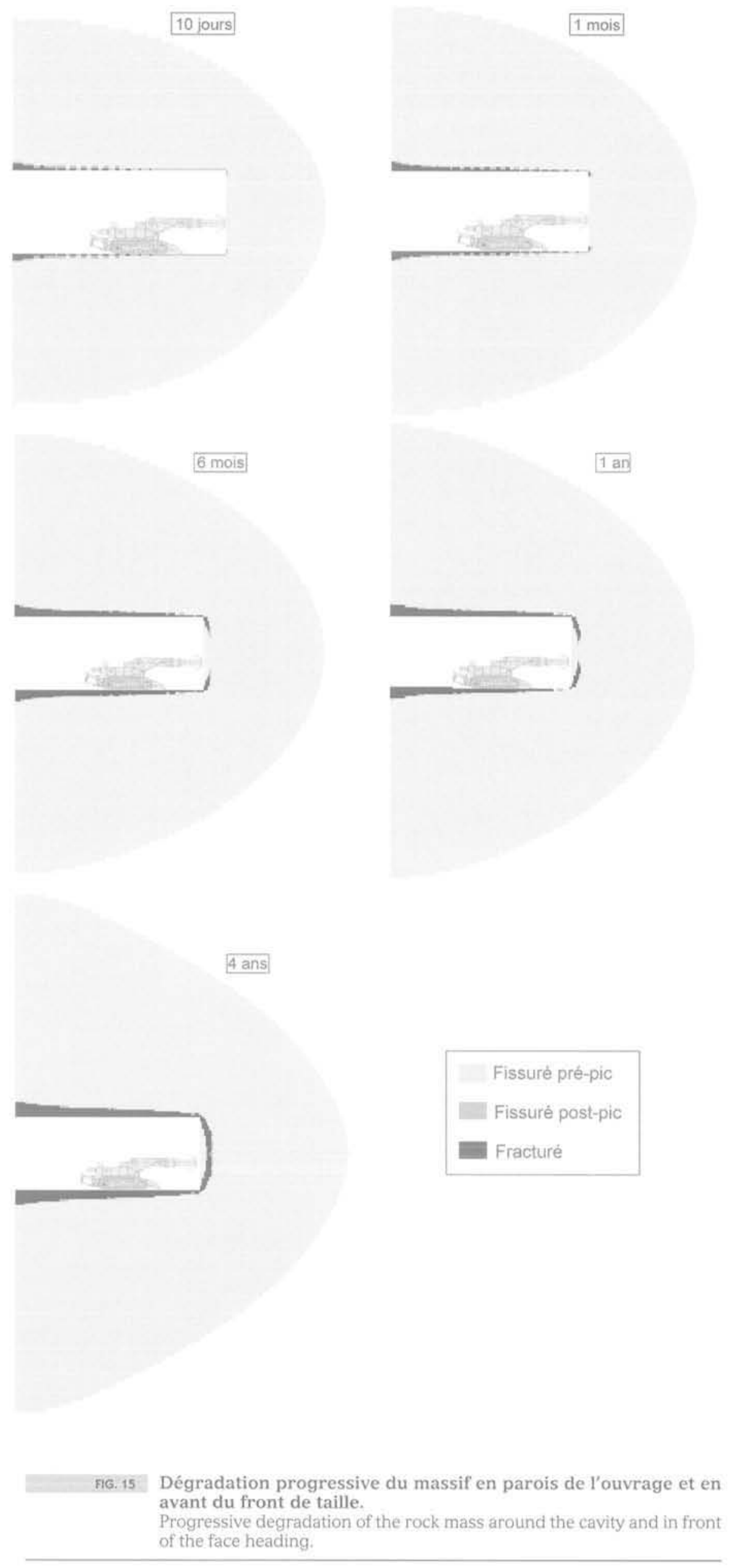


tibles de retarder le développement d'un mécanisme de ruine et d'effondrement. Par ailleurs, afin de garantir la sécurité du chantier et la stabilité à long terme du tunnel ou encore pour minimiser la zone fracturée, des structures de soutènement/revêtement seraient à envisager in fine.

Le scénario présenté ici consiste à poser un revêtement rigide (anneau de béton de $80 \mathrm{~cm}$ d'épaisseur) à une distance de $5 \mathrm{~m}$ du front de taille. La vitesse de creusement reste fixée à $2 \mathrm{~m} / \mathrm{j}$. Deux calculs ont été menés : l'un avec le modèle élastoplastique et l'autre avec le modèle viscoplastique. La figure 13 présente l'évolution de la contrainte maximale relevée dans le béton, pendant la phase d'excavation, dans chacun des cas, Les efforts prédits par le calcul viscoplastique sont presque deux fois plus élevés que ceux prédits par le calcul élastoplastique, à l'issue de la phase de creusement.

Contrairement à l'approche élastoplastique, l'approche viscoplastique permet d'analyser l'évolution des efforts dans le revêtement à moyen et long termes. La poursuite du calcul jusqu'à cent ans montre que les efforts continuent à augmenter après l'excavation, pour atteindre $22 \mathrm{MPa}$.

Par ailleurs, un accroissement de la vitesse de creusement peut s'accompagner d'une légère augmentation de la contrainte maximale locale de compression dans le béton.

Dans une étude d'ingénierie, l'utilisation d'un modèle élastoplastique est susceptible de conduire à un sous-dimensionnement des structures de soutènement/revêtement, ne pouvant garantir la stabilité à court terme du revêtement, ni la stabilité à long terme de l'ouvrage.

En terme de dégradation du massif, l'effet de la pose d'un revêtement sur l'index de fissuration est mis en évidence sur la figure 14. La mise en place du béton permet de réduire significativement la zone fracturée (de 17,5\%R à 2,5\%R avec la modélisation viscoplastique). De plus, l'effet du soutènement semble plus marqué avec l'approche viscoplastique.

\section{5}

\section{Comportement à long terme}

Les hypothèses des calculs présentés ci-dessous sont les mêmes que précédemment, sans mise en place de soutènement/revêtement. Après une excavation continue de $20 \mathrm{~m}$ de galerie, une interruption prolongée du chantier est simulée. Les graphiques présentés sur la figure 15 montrent l'évolution de la fracturation en paroi de la cavité et en avant du front taille, sur une période de quatre ans.

Ce mécanisme de fracturation, initié par la création d'un « coin » décomprimé, délimité par une surface de fracturation en cisaillement, est similaire au mécanisme bien connu qui se développe sous une semelle de fondation. Il peut être à l'origine d'une instabilité du front, en particulier sous l'effet de forces gravitationnelles non prises en compte dans ce cas.
De tels résultats sont aussi représentatifs d'un mécanisme de fracturation par chevron qui a été observé lors de l'excavation de certains tunnels dans des milieux argileux (CFMR, 2000).

\section{6}

\section{Conclusion}

La nature est complexe et c'est en toute modestie que les ingénieurs doivent chercher à prédire le comportement des ouvrages dans le sous-sol. La réalisation de projets industriels dans le domaine souterrain, à forts enjeux économiques et sociaux (traversées alpines, stockage de déchets nucléaires), nécessite d'évoluer vers une meilleure compréhension des mécanismes comportementaux des ouvrages à concevoir. Cette amélioration passe par une meilleure représentativité physique des mécanismes macroscopiques et par la mise à disposition d'outils de prédiction adaptés aux attentes et aux besoins des ingénieurs. Les outils de calculs récemment développés par EDF-CIH, dans le domaine de la conception des ouvrages souterrains, s'inscrivent dans cette volonté de rapprocher les attentes de l'industrie et les connaissances liées à la rhéologie des géomatériaux. Ces développements ont ainsi débouché sur la proposition de modèles de comportement mécaniques, adaptés aux roches peu fissurées et assimilables à des milieux continus, intégrant, en particulier, l'effet du temps.

Les outils de modélisation proposés, et la démarche d'interprétation associée, ont permis de montrer l'apport de la prise en compte du comportement différé sur la représentativité des prédictions numériques du comportement d'un tunnel à court, moyen et long termes. La viscosité des géomatériaux est généralement associée à la notion de long terme et, à ce titre. habituellement analysée de manière découplée du processus de conception à court terme. Or, il a été montré que le comportement différé a aussi une influence sur le comportement de l'ouvrage en cours de creusement. 11 convient donc d'intégrer des essais de fluage au stade du projet. Cependant, d'un point de vue expérimental, ce type d'essais reste difficile à mettre en ceuvre puisque la gamme des vitesses de sollicitation et l'amplitude des phénomènes induits sont de l'ordre de la limite de précision des appareils de mesure.

Ce mécanisme de comportement différé est un phénomène, parmi d'autres, qui justifie la différence de résistance apparente entre les observations en laboratoire et le retour d'expérience issu des ouvrages. D'autres phénomènes ne doivent cependant pas être négligés, en particulier les mécanismes de couplage hydromécaniques, qui peuvent avoir, dans le cas des roches argileuses, des temps caractéristiques du même ordre de grandeur que ceux du fluage/relaxation.

La représentativité du modèle rhéologique proposé icí, justifiée sur plusieurs ouvrages (Laigle, 2003), n'est pas seulement liée à la prise en compte du comportement différé, mais aussi à une formulation du comportement macroscopique de la roche adaptée dans le domaine des moyennes et grandes déformations (radoucissement). 
André D., Dardard B., Bouvard A. Carmes J. - La traversée des argiles du tunnel de Tartaiguille. Tunnels et Ouvrages souterrains, $n^{\circ} 153,1999$

Aubertin M., Simon R. - A damage initiation criterion for low porosity rocks. International Journal of Rock Mechanics and Mining Sciences, vol. 34, 3-4, 1997, paper $n^{\circ} 017$.

Aubertin M - A multiaxial stress criterion for short- and long-term strength of isotropic rock media. International Journal of Rock Mechanics and Mining Sciences, vol, 37, 2000, p. 1169-1193.

Bieniawski Z.T. - 1974. Estimating the Strength of Rock Materials. J.S. African. Institute of Mining and Metallurgy, 74 (8), 1974, p. 312-320

CFMR (Comité français de mécanique des roches) - Manuel de mécanique des roches. Presses de l'École des mines de Paris, 2000, tome 1.

Charmetton S. - Renforcement des parois d'un tunnel par des boulons expansifs. Retour d'expérience et études numériques. Thèse de l'Ecole centrale de Lyon, 2001.

Cho N., Christiansson R. - Suppressing fracture growth around underground opening, NARMS-TAC 2002, Hamma et al. (eds), University of Toronto, 2002.

Diederichs M.S, - Stress induced damage accumulation and implications for hard rock engineering. NARMS-TAC 2002 Hamma et al. (eds), University of Toronto, 2002.

Grgic D. - Modélisation du comportement à court et à long terme des roches de la formation terrifere de Lorraine. Thèse de I'INPL, 2001.

Helal H., Homand F., Josien J.-P. - Valiedity of uniaxial compression tests for indirect determination of long term strengti? of rocks. International Journal of Mining and Geological Engineering, vol. 6, 1988, p. 249-257.

Hoek E. Kaiser P.K. Bawden W.F. - Support of Underground Excavations in Hard Rock. A. A. Balkema, 1994

Laigle F, - A new Viscoplastic Model for Rocks: Application to the Mine-by-test of AECL-URL. Flac and Numerical Modeling in Geomechanics. Balkema, 2003. Laigle F.-Modèle conceptuel pour le développement de lois de comportement adaptées á la conception des ouvrages souterrains. Thèse de l'École centrale de Lyon, 2004.
Lau J.S.O., Chandler N.A. - Innovative laboratory testing. International Journal of Rock Mechanics and Mining Sciences, vol. 41, 2004, p. 1427-1445.

Kleine A. - Rapport d'avancement de thèse ( $2^{e}$ année), LAEGO-ENSG.LG.AK.EDF. PSI.RPRE.05.0087.A. 2006.

Martin C.D. Chandler N.A. - The Progressive Fracture of Lac du Bonnet Granite. Int. J. Rock. Mech. Min. \& Geomech. Abstr, n 31 (6), 1994, p. 643-659.

Perzyna P. - Fundamental Problems in Viscoplasticity. Advances in applied Mechanics, vol. 9, Academic Press New York and London, 1966.

Rowe P.W. - The Stress-Dilatancy Relationship for Static Equilibrium of an Assembly of Particles in Contact. Proceedings of Royal Society, vol. 269, 1962, p. $500-527$.

Schmidtke R.H., Lajtai E. Z. - The Longterm Strength of Lac du Bonnet Granite. International Journal of Rock Mechanics and Mining Sciences, vol. 22, 1985, p. 461-465.

Wiid B.L. - The influence of moisture on the pre-rupture fracturing of two rock types. Proc. 2nd Cong. Int. Soc. Rock Mech., Belgrade, vol. 2, 1970, p. 239-245. 